\title{
Subsurface Fractional Ablative Resurfacing of a Periareolar Scar Using 1,064-nm Picosecond Laser with Micro-Lens Array
}

\author{
Yeansu Choi, Eun Soo Park \\ Department of Plastic and Reconstructive \\ Surgery, Soonchunhyang Bucheon \\ Hospital, Soonchunhyang University \\ College of Medicine, Bucheon, Korea
}

This work was supported by the Soonchunhyang University Research Fund.

No potential conflict of interest relevant to this article was reported.

\begin{abstract}
Although picosecond lasers were invented as an effective treatment of pigmented lesions, the Food and Drug Administration has also approved picosecond lasers with fractional mode for treating acne scars and wrinkles on the face. In this report, we introduce a noteworthy case in which we treated a periareolar scar using a 1,064-nm picosecond laser with fractional mode. A 32-year-old female patient who had a periareolar scar due to a breast reconstruction procedure with a latissimus dorsi myocutaneous flap hoped to remove the postoperative scar. We applied a 1,064-nm picosecond neodymium-doped yttrium aluminium garnet (Nd:YAG) laser with a micro-lens array (MLA) to the scar twice, with a 1-month interval between treatments. The scar improved after the second laser treatment and the patient was satisfied with the outcome. The treatment of a postoperative periareolar scar through subsurface fractional ablative resurfacing using a 1,064-nm Nd:YAG picosecond laser with a MLA produced significant improvements in the texture and aesthetic appearance of the scar, without any wound complications.
\end{abstract}

Keywords Cicatrix, Laser therapy, Mammaplasty

\section{INTRODUCTION}

Picosecond lasers were first developed with the goal of enabling the more effective treatment of pigmented lesions, including tattoos. Recently, picosecond lasers with a focus lens array have been applied to facial wrinkles or scars [1,2]. In 2014, the Food and Drug Administration (FDA) approved a 755-nm picosecond alexandrite laser not only for the treatment of pigmented lesions and tattoos, but also for the treatment of scarring and wrinkles on the face.

Although slight differences exist between each fractional mode, depending on the laser device, such as the focus tip, diffractive lens

Received: Oct 17, 2017 Revised: Dec 6, 2017 Accepted: Dec 16, 2017 Correspondence: Eun Soo Park Department of Plastic and Reconstructive Surgery, Soonchunhyang University Bucheon Hospital, 170 Jomaru-ro, Wonmi-gu, Bucheon 14584, Korea. E-mail: peunsoo@schmc.ac.kr

Copyright () 2018 The Korean Society for Aesthetic Plastic Surgery.

This is an Open Access article distributed under the terms of the Creative Commons Attribution Non-Commercial License (http://creativecommons.org/licenses/by-nc/4.0/) which permits unrestricted non-commercial use, distribution, and reproduction in any medium, provided the original work is properly cited. www.e-aaps.org array (DLA), and micro-lens array (MLA), all handpieces have common structures, including a micro-convex lens concentrating the energy into each laser beam. This highly concentrated laser energy induces breakdown within the dermis, resulting in the increased production of new dermal collagen [3].

Therefore, picosecond lasers with fractional mode are a promising new option for scar management. Former studies have mainly focused on facial wrinkles and acne scars, and no studies have yet investigated the management of scars in areas other than the face with picosecond lasers. Herein, we describe the use of a 1,064-nm picosecond neodymium-doped yttrium aluminium garnet (Nd:YAG) laser with fractional mode to treat a periareolar scar in a patient who had undergone quadrantectomy and reconstruction of the left breast with a latissimus dorsi myocutaneous flap.

\section{CASE REPORT}

A 32-year-old female patient who was diagnosed with ductal carcinoma in situ in the left breast underwent a combined operation conducted by surgeons from the general surgery department and 

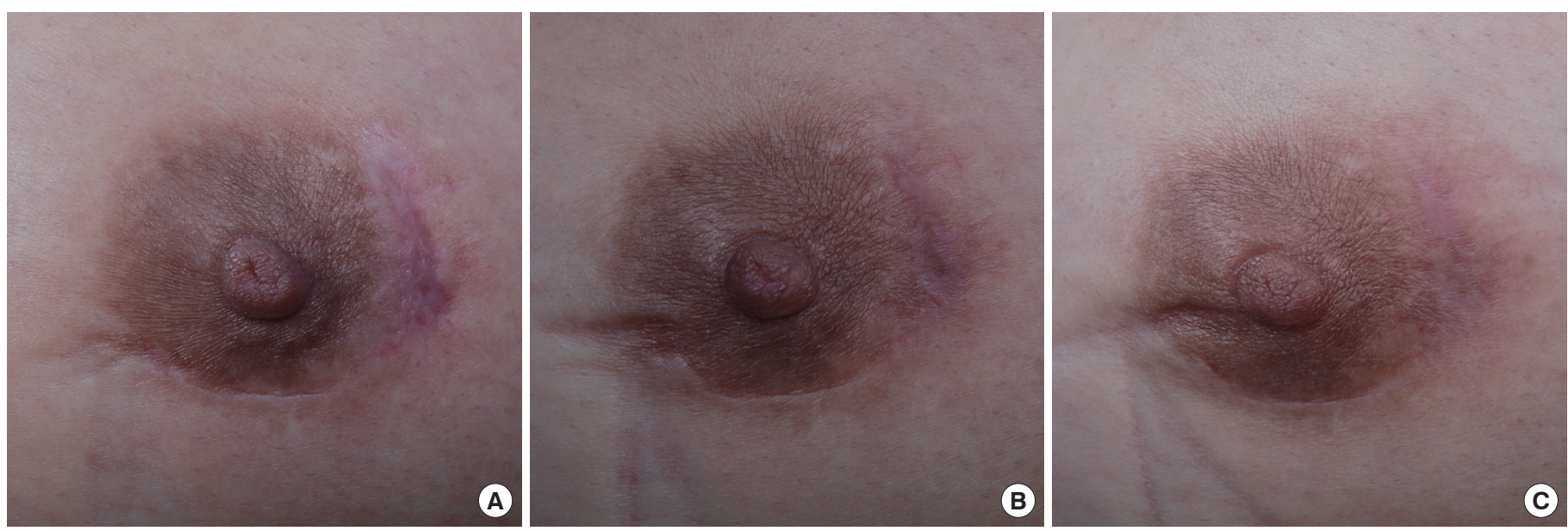

Fig. 1. (A) Before the first laser treatment. (B) A 1 month after the first laser treatment. (C) A 1 month after the second laser treatment.

plastic surgery department. The general surgery team removed the mass through a periareolar incision. After total resection of the mass, the plastic surgery team performed an immediate reconstruction of the breast with a latissimus dorsi myocutaneous flap. The patient was discharged on postoperative day 7 without any complications, such as wound dehiscence, infection, seroma, skin necrosis, or flap loss. We checked the flap viability and the progress of the postoperative wound every 3 days for a month, every week for 2 months, and then every month for 4 months in the outpatient department. No complications were found during the follow-up period, and the patient was satisfied with the overall outcome, except for a postoperative scar around the areola. The scar from the periareolar incision remained markedly visible, even 8 months after the operation.

A 1,064-nm picosecond Nd:YAG laser with fractional mode (PICOCARE $^{\circledR}$; WONTECH, Daejeon, Korea) was used to soften the scar. The patient received the laser treatments twice, at a 1-month interval. The first treatment was performed 8 months after surgery, with a spot size of $7 \mathrm{~mm}$, fluence of $1.2 \mathrm{~J} / \mathrm{cm}^{2}$, repetition rate of 1 $\mathrm{Hz}$, and pulse width of 750 picoseconds, in combination with an MLA. The second round of treatment was conducted with the same settings, and photographs were taken before each round of treatment.

At the end of the treatment, the patient was asked to rate her satisfaction with the improvements in the aesthetic appearance and texture of the scar using a 4-point scale ( 0 , not satisfied; 1 , dissatisfied; 2, satisfied; and 3, extremely satisfied). In addition, 1 plastic surgeon and 2 residents specializing in plastic surgery also evaluated the improvement of the patient's periareolar scar using a 4-point scale (0, 0-25\%; 1, 26-50\%; 2, 51-75\%; 3, 76-100\%) immediately after the second round of treatment and 1 month thereafter. The evaluators were not informed of which photo had been taken earlier.

The laser treatments using a picosecond laser with MLA were performed over the course of 2 months. After 2 picosecond laser treatment sessions, the periareolar scar appeared lighter and more natural (Fig. 1). No complications such as erythema, hyperpigmentation, or edema took place during the treatment period. The patient was satisfied with the aesthetic appearance of the scar and extremely satisfied with the texture after the final treatment, with a greater level of satisfaction than we expected. A masked evaluation of the photographs of the patient was performed by 3 evaluators. The mean scores were 1.66 immediately after the completion and 2.66 at 1 month thereafter, indicating that the scar had improved by at least $75 \%$ compared to its previous state.

\section{DISCUSSION}

Cosmetic laser treatments are in high demand among various patients with skin problems. The US FDA approved using 755-nm picosecond alexandrite lasers to remove tattoos in 2012, and they have been found to be effective for rapidly clearing unwanted tattoos, especially those with green and blue pigment [4]. Reiter et al. [5] conducted a large systematic review of the effect of picosecond lasers for tattoo removal. They concluded that picosecond lasers have a beneficial photoacoustic effect for multicolored tattoos, including green, yellow, red, and purple colors.

Picosecond lasers have attracted the attention of many plastic surgeons and dermatologists as a novel approach to a wide range of cosmetic problems of the skin, beyond just tattoos. Picosecond lasers with an ultra-short pulse duration were also approved by the FDA in 2014 for the treatment of wrinkles and acne scarring. Unlike existing laser treatments, whose mechanism is based on photothermolysis, picosecond lasers are believed to utilize energy from laser-induced optical breakdown (LIOB). The high energy delivered by the picosecond laser with a DLA targets melanin, which is located in the epidermal focal area. Melanocytes within the localized epidermal zone absorb the energy and generate an initial free 
electron. This free electron absorbs the laser light, effectively resulting in the generation of a second free electron. The cascade activation of surrounding electrons finally forms an ionized plasma that can absorb a large amount of the remaining laser radiation. The last step of LIOB is the formation of a vacuole in the epidermis when the plasma has absorbed sufficient energy. This injury within the epidermal layer created by a picosecond laser with a fractional lens array results in a rejuvenation effect, producing new dermal collagen, elastic tissue, and mucin [6].

In 2015, Brauer et al. [2] described favorable clinical outcomes in atrophic facial acne scars treated with a 755-nm picosecond laser with a DLA. They reported improvements in both the pigmentation and texture of the surrounding skin. Weiss et al. [7] in 2017 also performed a prospective study of 40 subjects, applying a 755$\mathrm{nm}$ picosecond laser with a novel DLA to signs of photoaging, including fine wrinkles. A blinded physician evaluated the improvement of the wrinkles, and the picosecond laser was found to be effective. Histologic data showing increased collagen and elastin also supported the textural improvements described by patients. A significantly reduced downtime was also identified as one of the most remarkable advantages of the picosecond laser.

In this case, the patient received 2 treatment sessions at a 4-week interval, and each treatment was tolerated well and performed safely. Clinical improvements were documented by subjective and objective evaluations, and no adverse effects took place. The patient was more satisfied with the outcomes than we expected. Additionally, she stated that she would recommend scar treatment using a picosecond laser to friends or family members with a similar scar.

Although a few studies have reported the use of a picosecond laser for scar management, our study is novel in several regards. First, in former studies, the treated lesions were acne scars and wrinkles located on the face. In our study, however, the scar was a postoperative wound located in the nipple-areolar complex. The periareolar region is anatomically unusual in the human body because it is the border of 2 types of skin that have different contours and colors from each other. Depressed and hyperpigmented periareolar scars can be elevated by the LIOB effect, with a dramatic outcome. Second, we applied a 1,064-nm picosecond Nd:YAG laser, whereas most previous studies used a 755-nm picosecond alexandrite laser. To our knowledge, this is the first report to describe the safety and efficacy of a 1,064-nm picosecond Nd:YAG laser with an MLA in the treatment of a linear scar in the periareolar region.

Our study is limited by the fact that we only described a single case of treatment and by the lack of an evaluation of histologic findings. However, many patients have similar scars after breast reconstruction operations performed following nipple-sparing mastectomy. We believe that picosecond lasers with a fractional mode have the possibility of being a more effective treatment modality than conventional lasers for depressed scars.

\section{PATIENT CONSENT}

Patient provided written consent for the use of her images.

\section{REFERENCES}

1. Khetarpal S, Desai S, Kruter L, et al. Picosecond laser with specialized optic for facial rejuvenation using a compressed treatment interval. Lasers Surg Med 2016;48:723-6.

2. Brauer JA, Kazlouskaya V, Alabdulrazzaq H, et al. Use of a picosecond pulse duration laser with specialized optic for treatment of facial acne scarring. JAMA Dermatol 2015;151:278-84.

3. Habbema L, Verhagen R, Van Hal R, et al. Minimally invasive nonthermal laser technology using laser-induced optical breakdown for skin rejuvenation. J Biophotonics 2012;5:194-9.

4. Brauer JA, Reddy KK, Anolik R, et al. Successful and rapid treatment of blue and green tattoo pigment with a novel picosecond laser. Arch Dermatol 2012;148:820-3.

5. Reiter O, Atzmony L, Akerman L, et al. Picosecond lasers for tattoo removal: a systematic review. Lasers Med Sci 2016;31:1397-405.

6. Tanghetti EA. The histology of skin treated with a picosecond alexandrite laser and a fractional lens array. Lasers Surg Med 2016;48:646-52.

7. Weiss RA, McDaniel DH, Weiss MA, et al. Safety and efficacy of a novel diffractive lens array using a picosecond $755 \mathrm{~nm}$ alexandrite laser for treatment of wrinkles. Lasers Surg Med 2017;49:40-4. 